\title{
Stabilizing stochastically-forced oscillation generators with hard excitement: a confidence-domain control approach
}

\author{
Irina Bashkirtseva ${ }^{1}$, Guanrong Chen $^{2}$ and Lev Ryashko ${ }^{1, a}$ \\ 1 Department of Mathematics, Ural Federal University, Lenina 51, 620083 Ekaterinburg, Russia \\ 2 Department of Electronic Engineering, City University of Hong Kong, Hong Kong, P.R. China
}

Received 20 June 2013 / Received in final form 6 September 2013

Published online 16 October 2013 - (c) EDP Sciences, Società Italiana di Fisica, Springer-Verlag 2013

\begin{abstract}
In this paper, noise-induced destruction of self-sustained oscillations is studied for a stochastically-forced generator with hard excitement. The problem is to design a feedback regulator that can stabilize a limit cycle of the closed-loop system and to provide a required dispersion of the generated oscillations. The approach is based on the stochastic sensitivity function (SSF) technique and confidence domain method. A theory about the synthesis of assigned SSF is developed. For the case when this control problem is ill-posed, a regularization method is constructed. The effectiveness of the new method of confidence domain is demonstrated by stabilizing auto-oscillations in a randomly-forced generator with hard excitement.
\end{abstract}

\section{Introduction}

Analysis of multi-stable nonlinear systems with coexisting different dynamical regimes is an important issue from both theoretical and practical points of view [1]. One of the classical examples of such systems is an electronic generator with hard excitement $[2,3]$. This model demonstrates two different dynamical regimes corresponding to two types of attractors: a stable equilibrium and a stable limit cycle. Depending on the initial state, this dynamical system either exhibits a transition to auto-oscillations or relaxes to an equilibrium. Examples of systems with a similar type of dynamics have been found in mechanics, chemical kinetics, biophysics, and so on.

It is well known that even small stochastic fluctuations, which inevitably present in any real nonlinear dynamical systems, may have unexpected noise-induced transitions and stochastic bifurcations [4-7]. As is well known, one reason for having such transformations is the multistability and high stochastic sensitivity of the coexisting deterministic attractors [8,9].

The stochastically-forced electronic generator with hard excitement is a typical and nontrivial example of such systems. In fact, for weak noise, oscillations in the operating mode of such a generator are disturbed only slightly. This, from a mathematical point of view, means that the randomly-forced trajectories are concentrated near a deterministic cycle as an etalon regime. As noise increases, these trajectories can escape with a high probability from

\footnotetext{
a e-mail: lev.ryashko@usu.ru
}

the basin of attraction of the stable limit cycle and, crossing the separatrix (unstable cycle), be localized at near the stable equilibrium for a long time. This phenomenon of a noise-induced suppression of auto-oscillations and destruction of the generator operating mode has been well known [10].

A similar phenomenon has been observed and studied for various dynamical models, in which an unstable limit cycle separates the basins of attraction of a stable equilibrium and a stable limit cycle [11-13].

A general theoretical approach based on the FokkerPlanck-Kolmogorov (FPK) equation gives a most detailed probabilistic description of stochastic dynamics. However, a direct use of this equation is technically difficult, even for two-dimensional nonlinear systems. Therefore, asymptotic methods and approximations are commonly used $[14,15]$.

On the other hand, controlling nonlinear systems with regular or chaotic oscillations has attracted a lot of attention in the last two decades [16,17]. Various approaches to controlling nonlinear stochastic dynamics are investigated, for instance, in [18]. One of the general ideas is to reshape the probability distribution function (pdf) by control input [19]. A stochastic control for noisy multistable systems was studied in [20].

Within the framework of the approach presented in this paper, the regulator parameters are determined with the help of the available analytical solutions of the FPK equation. Because this equation can be solved analytically only in limited cases, approximate methods are used to design the control $[21,22]$. For the case of an equilibrium, the 
approximation of pdf based on the SSF technique was used in [23] for stabilization and suppression of noise-induced chaos.

A case of a limit cycle is a subject of the presented paper. Here, the problem of the synthesis of the regulator for stabilizing the stochastic oscillations is considered.

For the approximation of pdf near the limit cycle, the SSF technique and confidence domain method are applied. Short mathematical background of this theory is presented in Appendix. In this paper, the stabilization problem for the randomly-forced limit cycle is reduced to the synthesis of narrow confidence bands using a small SSF.

In Section 2, a theory of feedback control of SSF is presented. A constructive description of the parameters that stabilize the regulator is precisely given for twodimensional systems. A case of two-dimensional control input, when the system is completely controllable, has been studied in [24]. In the presented paper, the case of onedimensional control input is investigated. In this realistic case, the considered problem of SSF synthesis is ill-posed. Here, a general method of the regularization is proposed.

In Section 2.1, a class of nonlinear stochastically-forced oscillators is considered. Here, it is shown that one can get a direct solution of this control problem without the additional regularization and derive explicit formulas for the feedback regulator.

In Section 3, the theoretical results are applied to the analysis of noise-induced destruction of the self-sustained oscillations in generator with hard excitement. Here, the SSF technique and new confidence domain method are used for the stabilization the operating mode of this generator.

\section{Control of stochastic cycles}

We focus on designing a feedback regulator, which stabilizes a cycle of the closed-loop deterministic system and synthesizes a required SSF (see Appendix) for the corresponding stochastic system:

$$
\dot{x}=f(x, u)+\varepsilon \sigma(x) \dot{w}(t) .
$$

Here $f(x, u)$ is a sufficiently smooth vector-function and $u$ is an $m$-dimensional vector of control parameters, and $w(t)$ is an $n$-dimensional standard Wiener process.

Assume that, for $\varepsilon=0$ and $u=0$, system (1) has a $T$-periodic solution $x=\xi(t)$ with a phase trajectory $\Gamma$ (cycle). The stability of $\Gamma$ is not assumed, however.

The stabilizing regulator shall be selected from the class $U$ of admissible feedbacks $u=u(x)$, satisfying the following conditions:

(a) $u(x)$ is sufficiently smooth and $\left.u\right|_{\Gamma}=0$;

(b) for the closed-loop deterministic system

$$
\dot{x}=f(x, u(x)),
$$

the solution $x=\xi(t)$ is exponentially stable in the neighborhood of the cycle $\Gamma$. Assume that the cycle $\Gamma$ of the deterministic system (2) is stabilizable, i.e. $U$ is not empty.
Here, the aim is to design the assigned stochastic sensitivity function $W$ for the randomly-forced control system (1).

For system (1), it follows from (A.2) that the matrix $W(t)$ is governed by

$$
\begin{aligned}
\dot{W}= & (F(t)+B(t) K(t)) W+W(F(t) \\
& +B(t) K(t))^{\top}+P(t) S(t) P(t),
\end{aligned}
$$

where

$$
\begin{aligned}
& F(t)=\frac{\partial f}{\partial x}(\xi(t), 0), \quad B(t)=\frac{\partial f}{\partial u}(\xi(t), 0), \\
& K(t)=\frac{\partial u}{\partial x}(\xi(t)), \quad S(t)=\sigma(\xi(t)) \sigma^{\top}(\xi(t)) .
\end{aligned}
$$

Abilities of controlling the sensitivity function $W(t)$ are completely determined by the first derivatives $\frac{\partial u}{\partial x}(\xi(t))$ of the function $u(x)$ but are independent of the higherorder terms. It allows to restrict the design, without loss of generality, by simpler regulators in the following feedback form:

$$
u=K(t(x)) \Delta(x),
$$

where $\Delta(x)=x-\gamma(x)$ is a deviation of the state $x$ from the deterministic cycle $\Gamma, \gamma(x)=\operatorname{argmin}_{y \in \Gamma}\|x-y\|$, $t(x)=\operatorname{argmin}_{t \in[0, T)}\|x-\xi(t)\|$.

Consider a set of $T$-periodic $m \times n$-matrices $\mathbf{K}=\{K \mid$ cycle $\Gamma$ of system (2), (4) is exponentially stable $\}$. It is assumed that $\mathbf{K}$ is not empty. Thus, the feedback matrix $K(t) \in \mathbf{K}$ completely determines the abilities of the regulator (4) to synthesize the SSF $W(t)$.

It follows from (3) that the problem of synthesis of the matrix $W(t)$ by the regulator (4) is reduced to the solution of the linear matrix equation

$$
\begin{aligned}
& B(t) K(t) W(t)+W(t) K^{\top}(t) B^{\top}(t)= \\
& \quad \dot{W}-F(t) W(t)-W(t) F^{\top}(t)-P(t) S(t) P(t) .
\end{aligned}
$$

In the case of $n=2$, it follows from (A.3) that the stochastic sensitivity function $\mu(t)$ of the cycle $\Gamma$ satisfies the boundary value problem

$$
\dot{\mu}=\left(a_{0}(t)+2 \beta^{\top}(t) k(t)\right) \mu+b(t), \quad \mu(0)=\mu(T),
$$

where

$$
\begin{aligned}
a_{0}(t) & =p^{\top}(t)\left[F^{\top}(t)+F(t)\right] p(t), \beta(t)=B^{\top}(t) p(t), \\
k(t) & =K(t) p(t), b(t)=p^{\top}(t) S(t) p(t) .
\end{aligned}
$$

The aim of the control is to synthesize the assigned SSF $\mu(t)$ for the cycle $\Gamma$ of the stochastic system (1).

Consider a set of admissible stochastic sensitivity functions:

$$
M=\left\{\mu \in C_{[0, T]}^{1} \mid \mu(t)>0, \quad \mu(0)=\mu(T)\right\} .
$$

A solution of the control problem for synthesizing the assigned SSF $\mu(t) \in M$ is reduced to the solution of the 
following system:

$$
\begin{gathered}
\beta^{\top}(t) k(t)=\alpha(t), \quad \alpha(t)=\frac{\dot{\mu}-a_{0}(t) \mu-b(t)}{2 \mu}, \\
K(t) p(t)=k(t) .
\end{gathered}
$$

A decision for this system relies on the properties of the function $\beta(t)$. If $\beta(t) \neq 0, t \in[0, T]$, then the system (8), (9) is solvable for any $\mu(t) \in M$. This case, when the cycle $\Gamma$ is completely stochastic controllable, has been studied in [24]. Note that, in this case, the matrix $B$ has to be quadratic and non-degenerate $(\operatorname{rank} B=2)$.

For many important controlled systems, the last condition is not fulfilled. For example, if the system has a single scalar control input $(m=1)$ then $\operatorname{rank} B \leq 1$, and the system $(8),(9)$ is not solvable for some $\mu(t)$.

In the presented paper, therefore, a general case is considered, when the function $\beta(t)$ possesses zero values at some points in the interval $[0, T]$. In this case, a decision for equation (8) is an ill-posed problem. To solve this equation, the most commonly used Tikhonov method of regularization [25] is applied. This method reduces the solution of equation (8) to the minimization of the following quadratic function:

$$
\left(\beta^{\top}(t) k(t)-\alpha(t)\right)^{2}+\delta\|k(t)\|^{2} \longrightarrow \min .
$$

Here, $\delta$ is a small positive regularization parameter. The problem (10) has a unique solution

$$
k(t)=\frac{\alpha(t)}{\beta^{\top}(t) \beta(t)+\delta} \beta(t) .
$$

From (7), (9) and (11), one gets the following equation for the feedback matrix $K(t)$ of the regulator (4):

$$
K(t) p(t)=\frac{\alpha(t)}{p^{\top}(t) B(t) B^{\top}(t) p(t)+\delta} B^{\top} p(t) .
$$

This equation is always solvable. Evidently, the matrix

$$
K(t)=\frac{\alpha(t)}{p^{\top}(t) B(t) B^{\top}(t) p(t)+\delta} B^{\top}
$$

is one of its solutions.

If the optimality condition $\|K(t)\|^{2} \longrightarrow$ min is required, then the unique solution of (12) can be found:

$$
K(t)=\frac{\alpha(t)}{p^{\top}(t) B(t) B^{\top}(t) p(t)+\delta} B^{\top} p(t) p^{\top}(t) .
$$

Consider the case of $m=1$, when the system (1) has only one scalar control input. In this case, $B(t)$ is a 2dimensional column vector-function, $\beta(t)=B^{\dagger}(t) p(t)$ is a scalar function,

$$
k(t)=\frac{\alpha(t) \beta(t)}{\beta^{2}(t)+\delta}
$$

in (11) is a scalar function, and the 2-dimensional row vector-function $K(t)$ in (13) can be written as:

$$
K(t)=k(t) p^{\top}(t) .
$$

In this case, the feedback (4) has an explicit representation:

$$
\begin{aligned}
u\left(x_{1}, x_{2}\right)= & k\left(t\left(x_{1}, x_{2}\right)\right)\left[p_{1}\left(t\left(x_{1}, x_{2}\right)\right)\left(x_{1}-\xi_{1}\left(t\left(x_{1}, x_{2}\right)\right)\right)\right. \\
& \left.+p_{2}\left(t\left(x_{1}, x_{2}\right)\right)\left(x_{2}-\xi_{2}\left(t\left(x_{1}, x_{2}\right)\right)\right)\right] .
\end{aligned}
$$

\subsection{Control of nonlinear stochastic oscillators}

Consider a nonlinear stochastic oscillator with control input:

$$
\ddot{x}=\varphi(x, \dot{x}, u)+\varepsilon \sigma(x, \dot{x}) \dot{w},
$$

where $\varphi$ and $\sigma$ are scalar functions, $w$ is a scalar Wiener process, and $\varepsilon$ is the noise intensity. Rewrite this equation (16) as a system:

$$
\begin{aligned}
& \dot{x}=y, \\
& \dot{y}=\varphi(x, y, u)+\varepsilon \sigma(x, y) \dot{w} .
\end{aligned}
$$

Assume that the uncontrolled deterministic system (17) (with $u=0, \varepsilon=0$ therein) has a $T$-periodic solution $(\bar{x}(t), \bar{y}(t))$.

For system (17), one has:

$$
\begin{gathered}
F=\left(\begin{array}{cc}
0 & 1 \\
\varphi_{x}^{\prime} & \varphi_{y}^{\prime}
\end{array}\right), \quad S=\left(\begin{array}{cc}
0 & 1 \\
0 & \sigma^{2}
\end{array}\right), \quad B=\left(\begin{array}{c}
0 \\
\varphi_{u}^{\prime}
\end{array}\right), \\
p=\frac{1}{\sqrt{\bar{y}^{2}+\varphi^{2}}}\left(\begin{array}{c}
-\varphi \\
\bar{y}
\end{array}\right), \\
a_{0}=p^{\top}\left(F+F^{\top}\right) p=\frac{2 \bar{y}}{\bar{y}^{2}+\varphi^{2}}\left(\bar{y} \varphi_{y}^{\prime}-\varphi\left(1+\varphi_{x}^{\prime}\right)\right), \\
b=p^{\top} S p=\frac{\bar{y}^{2} \sigma^{2}}{\bar{y}^{2}+\varphi^{2}}, \quad \beta=B^{\top} p=\frac{\bar{y} \varphi_{u}^{\prime}}{\sqrt{\bar{y}^{2}+\varphi^{2}}} .
\end{gathered}
$$

Here, matrices $F, S$, and vectors $p, B$ and scalars $a_{0}, b, \beta$ are $T$-periodic functions. The function $\varphi$ and its derivatives are calculated for $x=\bar{x}(t), y=\bar{y}(t), u=0$. In the above formulas, the dependence on $t$ is not indicated for simplicity.

Note that a limit cycle of the uncontrolled deterministic system (17) embraces an equilibrium $\left(x_{e}, 0\right)$ and intersects the $\mathrm{O} x$-axis at least at two points.

Consider a set $I=\{0 \leq t<T \mid \bar{y}(t)=0\}$.

For system (17), equation (8) can be written as:

$$
\beta(t) k(t)=\frac{\dot{\mu}(t)-a_{0}(t) \mu(t)-b(t)}{2 \mu(t)} .
$$

For $t \in I$, one has that $\beta(t)=0$, and (18) is not solvable for any $\mu \in M$. In Section 2.1 above, a method of regularization was applied to solve this problem. But due to the special structure of system (17), another method for solving equation (18) can be suggested. Indeed, the coefficients $\beta(t), a_{0}(t), b(t)$ have the following representations:

$$
\beta=\bar{y} \beta_{1}, \quad a_{0}=\bar{y} a_{1}, \quad b=\bar{y} b_{1},
$$


where

$$
\begin{aligned}
\beta_{1}=\frac{\varphi_{u}^{\prime}}{\sqrt{\bar{y}^{2}+\varphi^{2}}}, \quad a_{1} & =\frac{2}{\bar{y}^{2}+\varphi^{2}}\left(\bar{y} \varphi_{y}^{\prime}-\varphi\left(1+\varphi_{x}^{\prime}\right)\right), \\
b_{1} & =\frac{\bar{y} \sigma^{2}}{\bar{y}^{2}+\varphi^{2}} .
\end{aligned}
$$

Thus, equation (18) can be written as:

$$
\bar{y}(t) \beta_{1}(t) k(t)=\frac{\dot{\mu}(t)-\bar{y}(t) a_{1}(t) \mu(t)-\bar{y}(t) b_{1}(t)}{2 \mu(t)} .
$$

Consider a set $M_{1}=\left\{\mu \in M \mid \dot{\mu}(t) / \bar{y}(t) \in C_{[0, T]}\right\}$, and let $\mu(t) \in M_{1}$. For $\mu_{1}(t)=\dot{\mu}(t) / \bar{y}(t)$, it follows from $(20)$ that

$$
\beta_{1}(t) k(t)=\frac{\mu_{1}(t)-a_{1}(t) \mu(t)-b_{1}(t)}{2 \mu(t)} .
$$

Assume that $\varphi_{u}^{\prime} \neq 0$ and, therefore, $\beta_{1}(t) \neq 0$. Then, equation (21) has a unique solution:

$$
k(t)=\frac{\mu_{1}(t)-a_{1}(t) \mu(t)-b_{1}(t)}{2 \mu(t) \beta_{1}(t)} .
$$

In this case, the feedback (15) can be written in the following form:

$$
\begin{aligned}
u(x, y)= & k(t(x, y))\left[p_{1}(t(x, y))(x-\bar{x}(t(x, y)))\right. \\
& \left.+p_{2}(t(x, y))(y-\bar{y}(t(x, y)))\right] .
\end{aligned}
$$

For the cycle of system (17), the regulator (22), (23) provides an assigned stochastic sensitivity function $\mu(t) \in M_{1}$. It is worth noting that all constant stochastic sensitivity functions belong to $M_{1}$.

\section{Stabilization of stochastic oscillations in a generator with hard excitement}

Consider a stochastic system:

$$
\begin{aligned}
& \dot{x}=y, \\
& \dot{y}=\left(l+m x^{2}-x^{4}\right) y-x+\varepsilon \dot{w},
\end{aligned}
$$

where $w(t)$ is a scalar Wiener process. The deterministic system (24) (with $\varepsilon=0$ therein) is a well-known model for generator of auto-oscillations with hard excitation $[2,3,26]$.

This model demonstrates two types of attractors: an equilibrium and a limit cycle.

For $l<-m^{2} / 8$, the deterministic system has only a stable trivial equilibrium $\left(x_{e}=0, y_{e}=0\right)$. At $l=-m^{2} / 8$, a stable limit cycle is born through a saddle-node bifurcation. In the parameter range $-m^{2} / 8<l<0$, the model is bistable, possessing a stable equilibrium and a stable limit cycle separated by an unstable limit cycle. At $l=0$, the unstable limit cycle merges with the equilibrium through
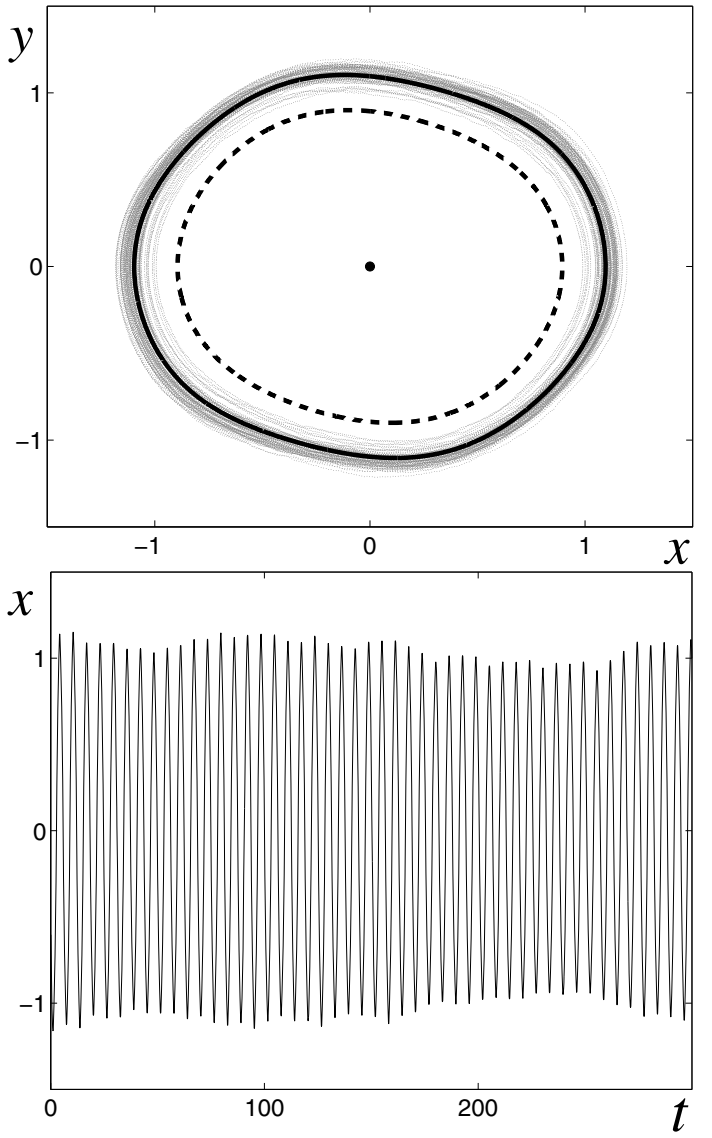

Fig. 1. (a) Stable (solid) and unstable (dashed) deterministic cycles, a stable equilibrium (circle) and random trajectories (grey color) for $\varepsilon=0.02$; (b) time series.

a subcritical Andronov-Hopf bifurcation. For $l>0$, the model has an unstable equilibrium and a stable limit cycle.

Oscillators coexisting with a stable equilibrium and a stable limit cycle, separated by an unstable cycle, are called "hard" because, for initial states arranged inside the unstable cycle, it is necessary to apply a strong excitation input to force the system to generate oscillations. In the present paper, the regime of hard excitation is studied for auto-oscillations at the fixed values $l=-0.12, m=1$. In Figure 1a, the trivial stable equilibrium $\left(x_{e}=0, y_{e}=0\right)$ (circle) and the stable limit cycle $\bar{x}(t), \bar{y}(t)$ (solid line), separated by the unstable limit cycle (dashed line), are all plotted.

Under stochastic disturbances, random trajectories of the forced system leave the deterministic cycle and form a kind of bundle around it. For weak noise, these trajectories with a high probability are localized near the unforced cycle and oscillations with small random variations of the amplitudes are observed. In Figure 1a, random trajectories of the system (24) with $\varepsilon=0.02$ are plotted in grey color. The corresponding time series for coordinate $x(t)$ is presented in Figure 1b.

As the noise intensity increases, random trajectories can escape from the basin of attraction of the limit cycle with a high probability, and then cross the separatrix 

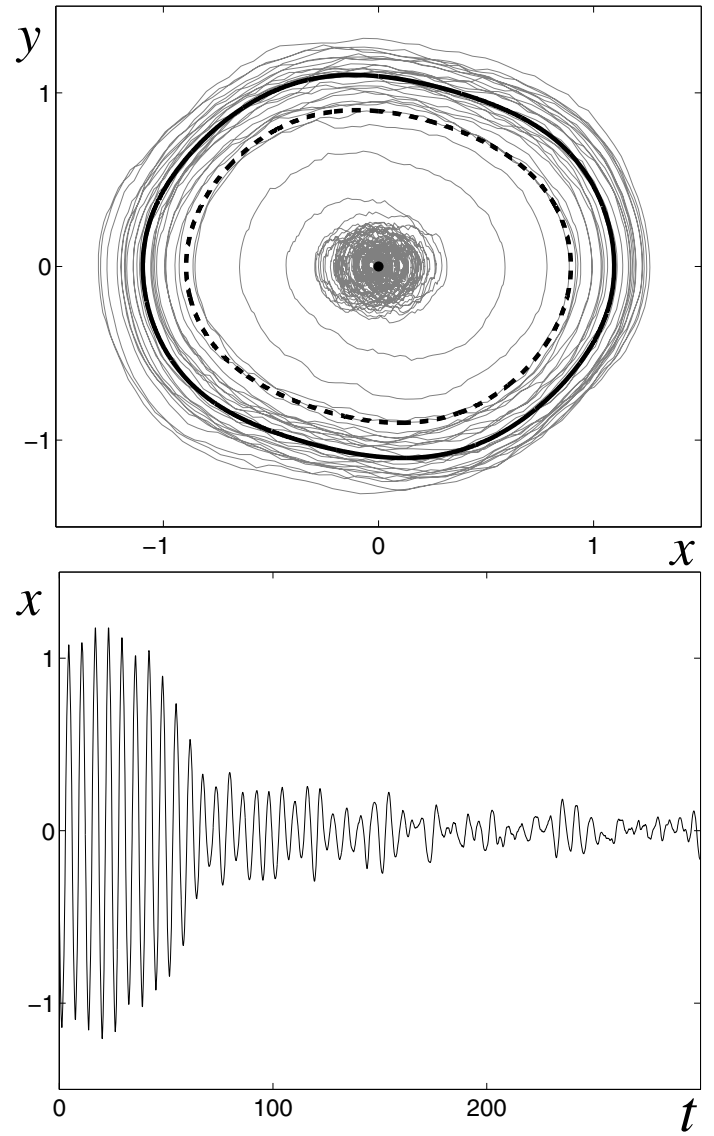

Fig. 2. (a) Stable (solid) and unstable (dashed) deterministic cycles, a stable equilibrium (circle) and random trajectories (grey color) for $\varepsilon=0.05$; (b) time series.

(unstable cycle), and eventually be localized near the stable equilibrium, as shown in Figure 2 for $\varepsilon=0.05$. So, here, noise suppresses auto-oscillations and destroys an operating mode of the generator.

To analyze this noise-induced phenomenon, the SSF technique and confidence domain method (see Appendix) are applied.

In Figure 3a, the SSF $\mu(t)$ is plotted. Using this function, one can construct the confidence band (A.4). In Figure $3 \mathrm{~b}$, the borders of the confidence band with fiducial probability $P=0.99$ for $\varepsilon=0.02$ are presented by dasheddotted lines. Here, the deterministic cycle (solid line) along with random trajectories (in grey color) are also shown. As can be seen, a spatial arrangement of the confidence band agrees with the dispersion of the random trajectories. The construction of the confidence band allows to analyze the phenomenon of the noise-induced suppression of oscillations without using the usual time-consuming direct numerical simulation of the stochastic trajectories.

A probabilistic mechanism responsible for this noiseinduced phenomenon can be explained by the mutual arrangement of confidence band and the separatrix (unstable cycle). In Figure 4, the deterministic cycle (solid line), separatrix (dashed line) and confidence band (in grey color) calculated via SSF technique are plotted for two values of the noise intensity. For weak noise $(\varepsilon=0.02)$,
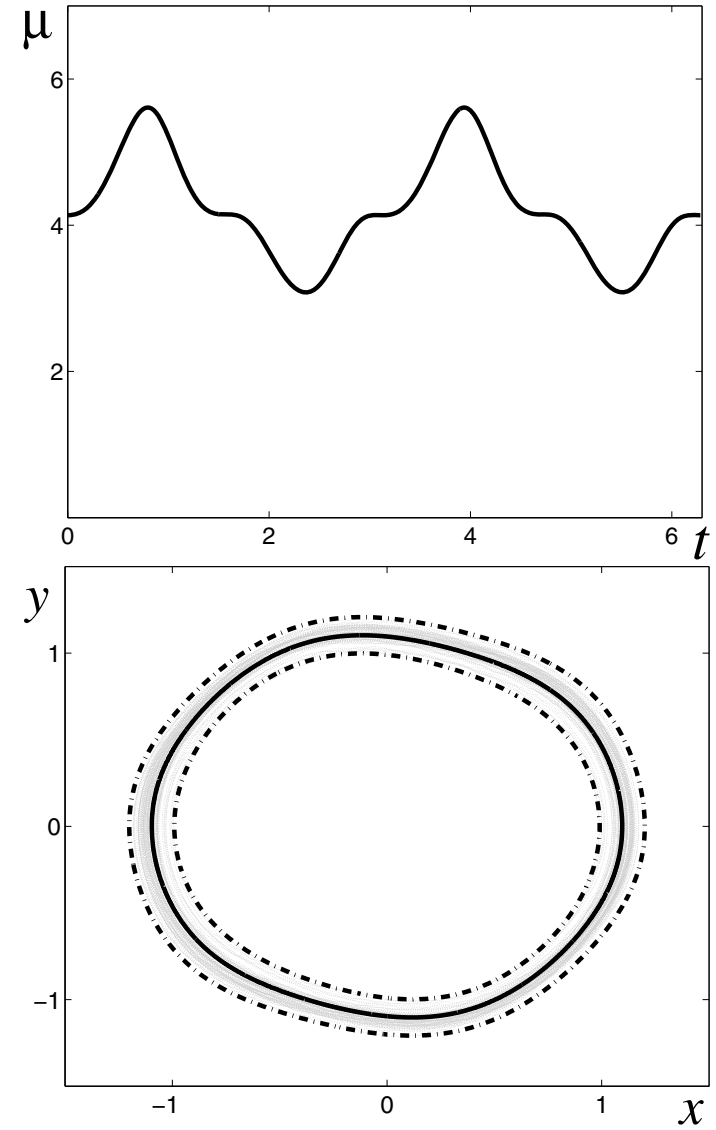

Fig. 3. (a) Stochastic sensitivity function; (b) random trajectories (grey color) and borders (dashed-dotted) of the confidence band for $\varepsilon=0.02, P=0.99$.

the confidence band entirely belongs to the basin of attraction of the stable cycle (see Fig. 4a). It means that random trajectories of the forced system oscillate with a small amplitude near the deterministic cycle (see Fig. 1 again). As the noise intensity increases (to $\varepsilon=0.05$ ), the confidence band expands across the separatrix and then begins to occupy the basin of attraction of the stable equilibrium (see Fig. 4b). This means that random trajectories starting near the stable limit cycle cross the separatrix and remain near the equilibrium with a high probability (see Fig. 2).

As a result, one can conclude that the method of confidence domain based on the SSF technique is a useful tool not only for the quantitative description of the dispersion of random trajectories, but also for detecting qualitatively the noise-induced deformations of the dynamics of this bistable system.

It should be noted that such a noise-induced deformation is an unwanted mode of the generator operation.

Furthermore below, it shows how the SSF technique can be applied for solving the stabilization problem of the noisy generator.

Consider a controlled stochastic system:

$$
\begin{aligned}
& \dot{x}=y \\
& \dot{y}=\left(l+m x^{2}-x^{4}\right) y-x+u+\varepsilon \dot{w}
\end{aligned}
$$



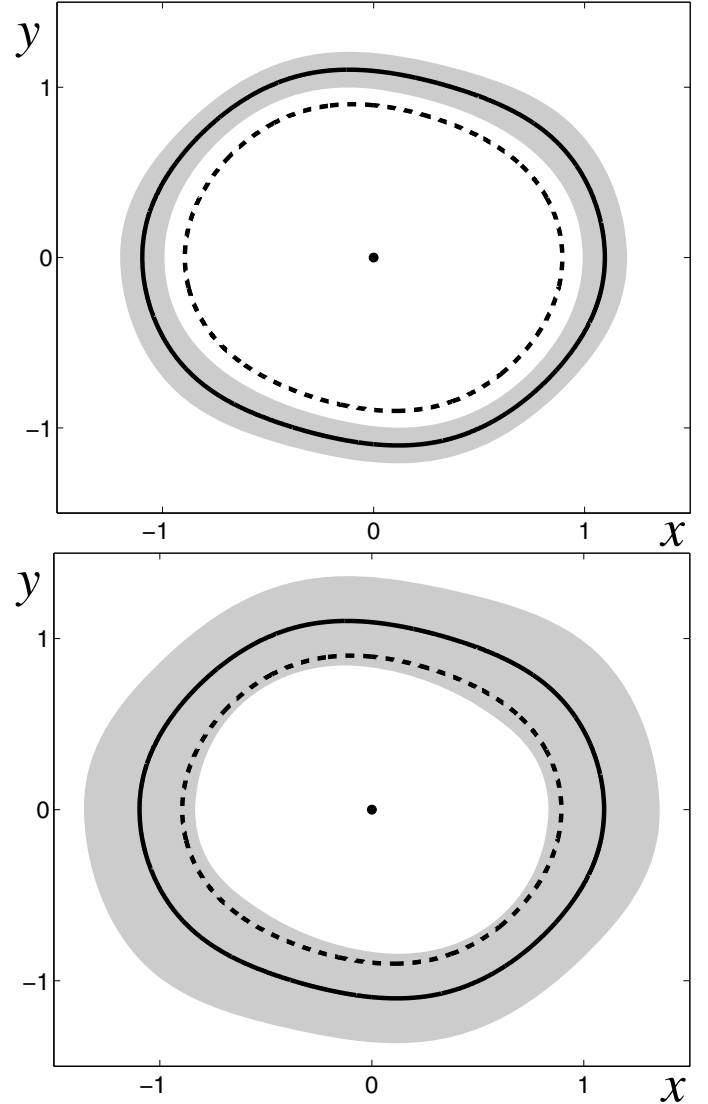

Fig. 4. (a) Stable (solid) and unstable (dashed) deterministic cycles, and confidence bands (grey color) with $P=0.99$ : (a) for $\varepsilon=0.02$; (b) for $\varepsilon=0.05$.

where $u$ is a scalar control input. To stabilize the noisy regulator, it is necessary to construct the feedback (23) in such a way as to localize a confidence band to be inside the basin of attraction of the cycle. As shown above, the confidence band for the uncontrolled system with $\varepsilon=0.05$ (see Fig. 4b) is too wide. Indeed, this confidence band contains the separatrix and partly occupies the basin of attraction of the equilibrium. It results in the noise-induced destruction of the operating mode of the generator (see Fig. 2). Here, the control problem has been reduced to decreasing the width of the confidence band.

Recall from Appendix that the width of the confidence band at the point $(\bar{x}(t), \bar{y}(t))$ of the cycle $\Gamma$ is given by the formula: $d(t)=2 q \varepsilon \sqrt{2 \mu(t)}$. In order to halve the value $d$, the value $\mu$ of the stochastic sensitivity should be reduced by four times. The SSF $\mu(t)$ of the uncontrolled system (24) changes (see Fig. 3a) within the interval $3<\mu(t)<6$. Set the SSF constant value $\mu(t) \equiv 1$ and construct the regulator (23) to provide this reduced value of the stochastic sensitivity for the closed-loop system (25), (23). For synthesizing this assigned SSF, the theory from Section 2.1 can be applied.

The regulator $(23)$ providing $\mu(t) \equiv 1$ compresses the confidence band and places it within the basin of attraction of the cycle, as expected. As a result, this regulator prevents unwanted noise-induced transition across the separatrix (see Fig. 5a).
A further improvement of the quality of the generator can be achieved by decreasing the stochastic sensitivity. Results from modeling the system with regulators providing $\mu=0.1$ and $\mu=0.01$ are presented in Figures 5b, $5 \mathrm{c}$, correspondingly. As can be seen, due to the reduction of the stochastic sensitivity, stabilization of oscillations forced by stochastic disturbances with $\varepsilon=0.05$ have been achieved.

The method developed in this paper can be applied to the stabilization of a wide class of randomly-forced generators with hard excitation. Indeed, from the mathematical standpoint, a peculiar feature of such generators is the coexistence of stable and unstable limit cycles.

Let $R$ be a distance apart from these cycles. By the SSF technique, the parametrical formula $r(t)=q \varepsilon \sqrt{2 \mu(t)}$ for the distance between the stable cycle and the border of the confidence band can be obtained. To provide the stability, one has to reduce the confidence band of the controlled system, so that this band does not intersect the unstable cycle. This geometrical condition of stability can be written as the inequality $\max _{[0, T]} r(t)<R$. This inequality implies the following condition:

$$
\max _{[0, T]} \mu(t)<\frac{R^{2}}{2 q^{2} \varepsilon^{2}} .
$$

The inequality (26) sets a relationship between the SSF $\mu(t)$, which is required for the stabilization, and the parameter $R$ of the deterministic system, as well as the value $\varepsilon$ of the noise intensity. To stabilize the oscillating mode of the generator, forced by noise with intensity $\varepsilon$, the proposed method is to first find an appropriate $R$ for the deterministic system, then choose $\mu$ to satisfy (26) (e.g., a constant), and finally apply the regulator to provide this value for $\mu$.

This work was partially supported by The Ministry of education and science of Russia (project 14.A18.21.0364) and RFBR (13-08-00069).

\section{Appendix: Background and the SSF technique}

Consider a nonlinear stochastic system in Ito's sense described by:

$$
\dot{x}=f(x)+\varepsilon \sigma(x) \dot{w},
$$

where $x$ is an $n$-vector, $f(x)$ is an $n$-vector function, $w(t)$ is an $n$-dimensional Wiener process and $\sigma(x)$ is an $n \times n$ matrix-valued function of disturbances with intensity $\varepsilon$. Assume that the corresponding deterministic system (A.1) (with $\varepsilon=0$ therein) has an exponentially stable limit cycle $\Gamma$ defined by a $T$-periodic solution $x=\xi(t)$.

Random trajectories of the forced system (A.1) leave a deterministic cycle and form a stochastic bundle with a stationary probability distribution $\rho(x, \varepsilon)$, which is a stationary solution of the corresponding Fokker-Planck equation. In general, to solve this equation is technically very difficult. For weak noise, asymptotics based on the quasipotential $v(x)=-\lim _{\varepsilon \rightarrow 0} \varepsilon^{2} \log \rho(x, \varepsilon)$ are commonly 

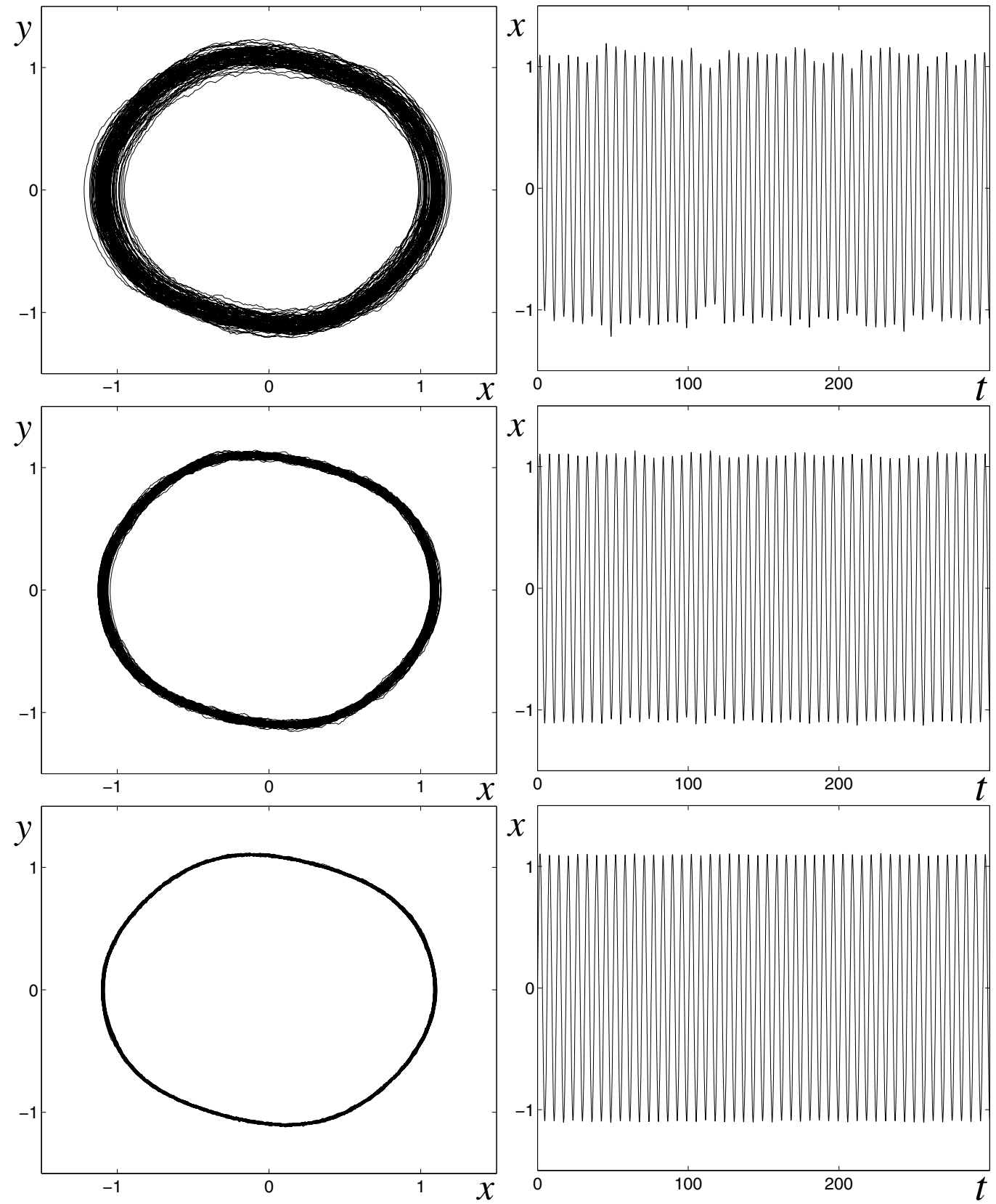

Fig. 5. Phase trajectories (left) and time series (right) for the stochastic system with $\varepsilon=0.05$ controlled by the regulator providing: (a) $\mu=1$; (b) $\mu=0.1$; (c) $\mu=0.01$.

used [15]. For weak noise, one can write an approximation of $\rho(x, \varepsilon)$ as follows:

$$
\rho(x, \varepsilon) \approx N \exp \left\{-\frac{v(x)}{\varepsilon^{2}}\right\} .
$$

In a neighborhood of the cycle $\Gamma$, a Gaussian approximation of the stationary probabilistic distribution for the Poincaré section $\Pi_{t}$ can be written as:

$$
\rho_{t}(x, \varepsilon) \approx N \exp \left\{-\frac{(x-\xi(t))^{\top} W^{+}(t)(x-\xi(t))}{2 \varepsilon^{2}}\right\}
$$

Here, $\Pi_{t}$ is a hyperplane orthogonal to the cycle at the point $\xi(t), 0 \leq t<T$. The stochastic sensitivity matrix $W(t)$ of the cycle is a unique solution of the Lyapunov equation [27]

$$
\dot{W}=F(t) W+W F^{\top}(t)+P(t) S(t) P(t),
$$

with conditions

$$
W(0)=W(T), \quad W(t) f(\xi(t)) \equiv 0,
$$

where $F(t)=\frac{\partial f}{\partial x}(\xi(t)), S(t)=\sigma(\xi(t)) \sigma^{\top}(\xi(t))$, and $P(t)$ is a matrix of the orthogonal projection on to the hyperplane $\Pi_{t}$. 
For the case of $n=2$, the stochastic sensitivity matrix $W(t)$ can be written as $W(t)=\mu(t) P(t)$. Here, $\mu(t)>0$ is a $T$-periodic scalar stochastic sensitivity function satisfying the following boundary problem [28]:

$$
\dot{\mu}=a(t) \mu+b(t), \quad \mu(0)=\mu(T),
$$

with $T$-periodic coefficients

$$
a(t)=p^{\top}(t)\left(F^{\top}(t)+F(t)\right) p(t), \quad b(t)=p^{\top}(t) S(t) p(t),
$$

where $p(t)$ is a normalized vector orthogonal to $f(\xi(t))$. The explicit solution $\mu(t)$ of the boundary problem (A.2) is given by:

$$
\mu(t)=g(t)(c+h(t))
$$

where

$$
\begin{gathered}
g(t)=\exp \left\{\int_{0}^{t} a(s) d s\right\}, \quad h(t)=\int_{0}^{t} \frac{b(s)}{g(s)} d s, \\
c=\frac{g(T) h(T)}{1-g(T)} .
\end{gathered}
$$

Using the stochastic sensitivity function $\mu(t)$, one can construct a confidence band around the deterministic cycle. The SSF $\mu(t)$ characterizes a spatial arrangement and size of the stationary distributed random state of the stochastic system (A.1) around the deterministic cycle.

For the line $\Pi_{t}$ that is orthogonal to the cycle at the point $\xi(t)$, the corresponding confidence interval is given by the following equation:

$$
(x-\xi(t))^{2}=2 q^{2} \varepsilon^{2} \mu(t) .
$$

Here, the parameter $q$ is connected with the fiducial probability $P$ by the formula $q=\operatorname{erf}^{-1}(P)$, where $\operatorname{erf}(x)=$ $\frac{2}{\sqrt{\pi}} \int_{0}^{x} e^{-t^{2}} d t$ is the error function.

Thus, the boundaries $x_{1,2}(t)$ of the confidence band can be written in an explicit parametrical form, as:

$$
x_{1,2}(t)=\xi(t) \pm q \varepsilon \sqrt{2 \mu(t)} p(t),
$$

and the width $d(t)$ of the confidence band at the point $\xi(t)$ of the cycle $\Gamma$ is given by the formula $d(t)=2 q \varepsilon \sqrt{2 \mu(t)}$. As can be seen, for the fixed value of the fiducial probability, this width is proportional to the noise intensity $\varepsilon$ and also to $\sqrt{\mu(t)}$. So, the SSF $\mu(t)$ provides a simple parametric description of the spatial probabilistic distribution of the random trajectories in a stochastic bundle by the confidence domain.

In a controlled system, one can change $\mu(t)$ by the variation of the regulator parameters. Therefore, one can change a width of the confidence domain and control the spatial probabilistic distribution of random states near the cycle. Constructive abilities of such approach are discussed in Sections 2, 3 of this paper.

\section{References}

1. J. Guckenheimer, P. Holmes, Nonlinear Oscillations, Dynamical Systems, and Bifurcations of Vector Fields (Springer-Verlag, New York, 1983), p. 484

2. A.A. Andronov, A.A. Vitt, S.E. Khaikin, Theory of Oscillators (Pergamon Press, Oxford, 1966)

3. P.S. Landa, Regular and Chaotic Oscillations (Springer, Berlin, 2001)

4. W. Horsthemke, R. Lefever, Noise-Induced Transitions (Springer, Berlin, 1984)

5. F. Moss, P.V.E. McClintock, Noise in nonlinear Dynamical Systems (Cambridge University Press, Cambridge, 1989)

6. L. Arnold, Random Dynamical Systems (Springer-Verlag, Berlin, 1998)

7. V.S. Anishchenko, V.V. Astakhov, A.B. Neiman, T.E. Vadivasova, L. Schimansky-Geier, Nonlinear Dynamics of Chaotic and Stochastic Systems. Tutorial and Modern Development (Springer-Verlag, Berlin/Heidelberg, 2007)

8. F. Arecchi, R. Badii, A. Politi, Phys. Rev. A 32, 402 (1985)

9. S. Kraut, U. Feudel, Phys. Rev. E 66, 015207 (2002)

10. R.L. Stratonovich, P.S. Landa, Izv. vuzov, Radiofizika 2, 37 (1959) (in Russian); English translation: in Non-Linear Transformations of Stochastic Processes, (Pergamon Press, Oxford, 1965), pp. 259-268.

11. A.L. Kawczynski, B. Nowakowski, Phys. Chem. Chem. Phys. 10, 289 (2008)

12. A. Zakharova, T. Vadivasova, V. Anishchenko, A. Koseska, J. Kurths, Phys. Rev. E 81, 011106 (2010)

13. X. Yue, W. Xu, L. Wang, B. Zhou, Probabilistic Engineering Mechanics 30, 70 (2012)

14. R.L. Stratonovich, Topics in the Theory of Random Noise (Gordon and Breach, New York, 1963)

15. M.I. Freidlin, A.D. Wentzell, Random Perturbations of Dynamical Systems (Springer, New York, 1984)

16. G. Chen, X. Yu, Chaos Control: Theory and Applications (Springer-Verlag, New York, 2003)

17. A.L. Fradkov, A.Yu. Pogromsky, Introduction to Control of Oscillations and Chaos, World Scientific Series of Nonlinear Science (World Scientific Publishing, 1998)

18. J.-Q. Sun, Stochastic Dynamics and Control (Elsevier, Amsterdam, 2006)

19. O. Elbeyli, J.-Q. Sun, J. Vib. Acoust. 126, 71 (2004)

20. B.E. Martinez-Zerega, A.N. Pisarchik, Commun. Nonlinear Sci. Numer. Simulat. 17, 4023 (2012)

21. L.A. Socha, M.J. Blachuta, in Proceedings of the American Control Conference 4, 2775 (2000)

22. L. Guo, H. Wang Stochastic Distribution Control System Design: A Convex Optimization Approach (SpringerVerlag, New York, 2010)

23. I. Bashkirtseva, G. Chen, L. Ryashko, Commun. Nonlinear Sci. Numer. Simulat. 17, 3381 (2012)

24. I. Bashkirtseva, L. Ryashko, Chaos Solitons Fractals 26, 1437 (2005)

25. A.N. Tikhonov, V.Y. Arsenin, Solution of Ill-posed Problems (Winston \& Sons, Washington, 1977)

26. Yu.I. Neimark, Soft and hard regimes of exciting autooscillations, in Mathematical Models in Natural Science and Engineering (Foundations of Engineering Mechanics, Springer, 2003), pp. 197-203

27. I.A. Bashkirtseva, L.B. Ryashko, Math. Comput. Simul. 66, 55 (2004)

28. I.A. Bashkirtseva, L.B. Ryashko, Physica A 278, 126 (2000) 